\title{
Internal Derangement of Temporomandibular Joint - A Review
}

\author{
Sharmila devi Devaraj ${ }^{1}$, Dr. Pradeep D ${ }^{2}$ \\ ${ }^{I}$ Final Year BDS, Saveetha Dental College and Hospitals, Saveetha University, Chennai, India. \\ 2Senior lecturer in Oral Maxillofacial and Surgery, Saveetha Dental College and Hospitals, \\ Saveetha University, Chennai, India.
}

\begin{abstract}
Temporomandibular joint (TMJ) is a complex, sensitive, and highly mobile joint. Temporomandibular disorders (TMD) are a class of degenerative musculoskeletal conditions associated with morphologic and functional deformities that affect up to $25 \%$ of the population. The most frequent structural (as opposed to muscular) cause of TMD are Internal derangement, which involves progressive slipping a displacement of component of temporo mandibular joint called articular disc.The disc is a piece of cartilage located between the condyle (ball) and fossa (socket)of the joint.The disc can degenerate, becoming misshapes or even torn. Because the deranged joint willcontinue to try to function in an impaired manner, so that internal derangement disorder often get progressively worse with time. In the mechanically demanding and biochemically active environment of the TMJ, therapeutic conservative and surgical approaches that can restore joint functionality while responding to changes in the joint have become a necessity.
\end{abstract}

Keywords: Temporomandibular joint (TMJ), Internal disc derangement, Oro facial pain, Temporomandibular joint (TMJ) disorder.

\section{Introduction}

Internal derangement of the temporo mandibular joint (TMJ) is defined as a disruption within the internal aspects of the TMJ in which there is a displacement of the disc from its normal functional relationship with the mandibular condyle and the articular portion of the temporal bone.[1] Internal derangement of the temporomandibular joint (TMJ) is characterized by displacement of the intra-articular disc, results in clicking or popping sounds. The displacement of the articular disc does not always cause a mechanical obstruction .[2] Those conditions may be painless or may be associated with pain, especially during function. The most common causes are trauma, which results in a sudden displacement of the disc, or chronic para function, which ends up in degenerative changes in the articular surfaces, increased friction, and gradual disc displacement.[2]

Farrar has estimated that up to $25 \%$ of the entire population has an internal derangement, which is initially treated with non-surgical method.[3] When these methods does not provide any of good prognosis, they are often followed by surgical methods such as meniscectomy, disc repositioning and disc reconstruction procedures and condylotomy. In the past, arthroscopic surgery and recently, arthrocentesis have been used with increasing frequency to treat TMJ internal derangements that fail to improve following a reasonable course of non-surgical therapy. Recent advance development in treatment includes intra injection of corticosteroids ,low level laser, tissue engineering techniques.

\section{Anatomy Of Temporomandibular Joint}

The temporomandibular joint is the articulation between the mandible and the cranium. The

mandibular head (condyle), glenoid (mandibular) fossa, and articular eminence form the TMJ. These joints serve as one anatomic control for both mandibular movement and the occlusion, surrounded by a capsule which consists of fibrous material, and a synovial lining. TMJ is reinforced by the temporo mandibular and spheno mandibular ligaments. The articular surface of the mandible is the upper and anterior surface of the condyle are lined by dense, avascular fibrous connective tissue. An articular disc is interposed between the temporal bone and the mandible that divides the articular space into upper and lower compartments. In a physiologic joint, the disc is positioned between the mandibular head inferiorly and the articular eminence anteriorly and superiorly when the jaw is closed. When the jaw is opened, the disc slides into a position between the mandibular head and articular eminence. The attachments of the disc prevent luxation during opening. A triangular lateral ligament acts as a strong lateral stabilizer and inhibits the posterior translation of the mandibular head. 


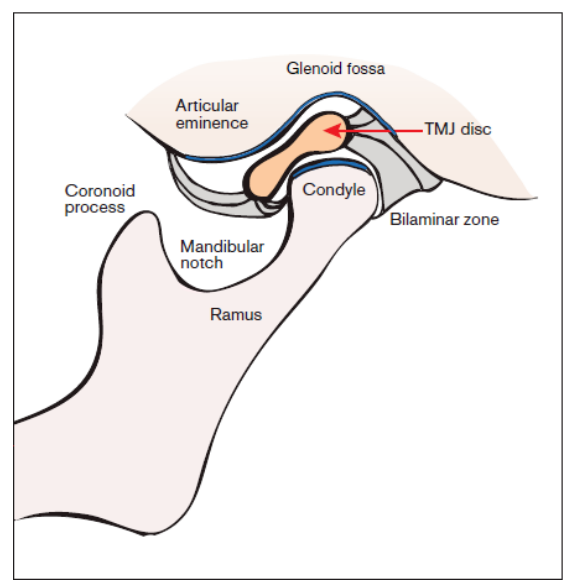

Fig 1 Sagittal view of temporo mandibular joint

The muscles of mastication are responsible for the complex movement of the jaw. The temporal, medial pterygoid, and masseter muscles which facilitates the jaw closure. Mouth opening is by coordinated muscular action of the lateral digastric, mylohyoid, and supra hyoid muscles. The lateral pterygoid muscle and part of fibers of the masseter and medial pterygoid muscles effect the anterior translation of the mandible. The superior belly plays a key role in the physiologic position of the disc as it pulls the disc forward when the jaw is opened, in a combined translation and rotation motion. The blood supply to the TMJ by temporal superficial artery, superior auricular artery, anterior tympanic artery and pterygoid artery. Nerve supply is provided by the auriculotemporal nerve (sensory branch of the mandibular nerve), deep temporal nerve, masseteric nerve. Sensory cervical sympathetic ramifications supply to the disc and capsule. [4]

\section{Tmj Disorder}

Temporomandibular disorder (TMD) is a generic term used for any problem concerning the jaw joint. Injury to the jaw, temporo mandibular joint or muscles of the head and neck may cause TMD. Other causes includes clenching of teeth, in which pressure on the TMJ induced ; dislocation of the disc; presence of osteoarthritis or rheumatoid arthritis in the TMJ; stress, aging. The most common TMJ disorders are pain dysfunction syndrome, internal derangement, arthritis, and traumas.TMD is seen most commonly in people between the ages of 20 and 40 years, and occurs more often in women than in men [5]. The most frequent complaint is pain and a decrease in the maximal interincisal opening (MIO), which normal values are between $35-50 \mathrm{~mm}$.The following symptoms as pain at rest, during maximum mouth opening and chewing, tenderness to palpation of the joint, sounds are clicking, crepitation, difficulty in opening the mouth, intermittent lock, closed lock, stiffness of joint in early morning are noticed .[4]

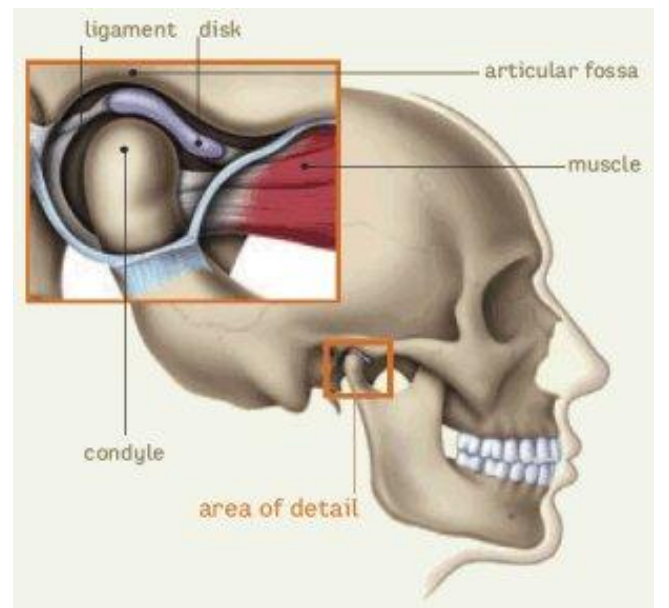

Fig 2 Anatomic structure of temporo mandibular joint 


\section{Classification}

Wilkes classification of internal derangement of TMJ [6]

\begin{tabular}{|c|c|c|}
\hline STAGE & CLINICAL & RADIOGRAPHIC \\
\hline I-EARLY & $\begin{array}{l}\text { Painless clicking; no limitation } \\
\text { of opening }\end{array}$ & $\begin{array}{l}\text { Mild disk displacement with } \\
\text { early reduction; normal disk } \\
\text { morphology }\end{array}$ \\
\hline II-EARLY/INTERMEDIATE & $\begin{array}{l}\text { Occasional painful clicking, } \\
\text { intermittent locking }\end{array}$ & $\begin{array}{lrr}\text { Mild to moderate } & \text { disk } \\
\text { displacement } & \text { with } & \text { late } \\
\text { reduction, mild disk deformity }\end{array}$ \\
\hline III-INTERMEDIATE & $\begin{array}{l}\text { Joint tenderness ,limited mouth } \\
\text { opening, frequent pain }\end{array}$ & Displaced, non reducing disk \\
\hline IV-INTERMEDIATE /LATE & Chronic pain,limited opening & $\begin{array}{l}\text { Severe displacement without } \\
\text { reduction, degenerative osseous } \\
\text { change }\end{array}$ \\
\hline V-LATE & $\begin{array}{l}\text { Variable joint } \\
\text { crepitus }\end{array}$ & $\begin{array}{l}\text { Non reduction of disk with } \\
\text { perforation of disk attachment } \\
\text { or disk degenerative osseous } \\
\text { changes. }\end{array}$ \\
\hline
\end{tabular}

\section{Aetiology}

Mostof the scientists explains that osteoarthritis as an inflammatory process, being most frequent one is the TMJ disorder, characterised by proliferative changes in the synovial and primary degeneration of the cartilage and surrounding tissues.[8,9] It is found that $28 \%$ of the adult population have symptoms and clinical signs of temporo mandibular joint disorder.[10,11] The aetiological factors of TMJ disorders as follows: systemic diseases ( rheumatoid arthritis, psoriasis, pseudogout, ankylosing spondylitis, etc.), secondary inflammatory component from the neighbouring regions (otitis, maxillary sinusitis, tonsillitis), trauma (chronical), prevalence of dental arch defects e.g. missing of molar teeth[12], malocclusion, endocrinological disturbances, odontogenic infections ( impacted third molars). Presence of specific bacterial organisms such as Staph.aureus, Strep. mitis, M. fermentas, Actinobacillus actinomycetemcomitans in the synovial fluid have been found .[13] Serum antibodies against Chlamydia species in patients with mono arthritis of the TMJ have also been reported[14]

\section{Pathogenesis}

Inflammation mainly affects the posterior disc attachment .[8,15] Several inflammatory mediators play an important role in the pathogenesis of TMJ disorders like tumor necrosis factor alpha (TNFalpha), interleukin-1beta (IL-1beta), prostaglandin E2 (PGE2), leukotriene B4 (LkB4 ), matrix metalloproteinases (MMPs), serotonin-5-hydroxytryptamine (5-HT).[10,16] MMPs are the early marker or detector to determine temporo mandibular joint arthritis. [17]. Serotonin is the mediator of pain and inflammation is produced in enterocromaffin cells of the gastrointestinal mucosa and absorbed by platelets. It is also produced in the synovial membrane and in the synovial fluid which causes TMJ pain in cases of systemic inflammatory joint diseases . $[18,19]$ Inflammation results in tissue response as : vasodilatation, extra vasation, release of mediators, activation of nociceptors, release of neuro peptides as substance P (SP), neuropeptide Y (NPY), which stimulate release of inflammatory mediators like histamine and serotonin and hyperalgesia.

\subsection{History and Physical Examination}

\section{Evaluation And Diagnosis}

Complete History taking from the patient, head and neck evaluation, general physical examination and clinical examination are essential .

\subsection{Laboratory Studies}

Laboratory studies are rarely indicated for ID/OA. In other suspected conditions of the TMJ, for example rheumatoid arthritis, the appropriate tests should be ordered.

\subsection{Imaging}

Imaging of the temporomandibular joints and associated structures is necessary to establish the presence or absence of pathology and stage of disease in order to select the appropriate treatment, assist in prognosis, and to assess patient response to therapy. Imaging results will influence treatment strategy.

\subsection{Radiographs}

This can provide the information include plain films, panoramic films, and tomograms (frontal and lateral) . The disc and associated soft tissue structures should also be imaged by Magnetic resonance imaging (MRI) [20,21] or arthrography.[22] Other radiological studies may also be indicated. 


\subsection{Computed tomography (CT)}

It is very useful to assess bone abnormalities like ankylosis, dysplasia, growth abnormalities, fractures, osseous tumors. [23] 3-D CT is a valuable diagnostic advancement for complex cases needing major reconstructive surgery. A stereo lithography model of a patient's maxillofacial skeleton can be fabricated utilizing 3-D CT technology .[23,24]

\subsection{Magnetic Resonance Imaging}

MRI is used to assess soft tissues, bone marrow changes, disc position, morphology, mobility, and joint effusion. [25,26] Cine MRI has been used to study the static disc or anchored disc.

\subsection{Arthrography}

MRI has largely replaced by arthrography [23] as the primary imaging study for the pathology of the disc .

\subsection{Isotope Bone Scan}

Radioactive isotope bone scans have a high sensitivity for detecting metabolic activity and

inflammation. [27] Single Photon Emission Computed Tomography (SPECT) is a form of isotope imaging utilizes the computer techniques to improve visualization of the plane of interest.

\section{8. Treatment}

An internal derangement TMJ are treated with nonsurgical methods which includes medications, physiotherapy and occlusal splints in the initial stages. [28]

\subsection{Non Invasive Methods}

Non surgical methods such as modification of the diet, occlusal splints, physical therapy, pharmacotherapy, transcutaneous electrical nerve stimulation (TENS) and stress reduction technique followed by surgical methods such as arthroscopy, reconstruction arthroplasty (disk repositioning), meniscectomy, and repair of perforation of disk. [29,30] When these methods are unsuccessful, they are managed by surgical methods. The surgical treatment is based on change of the morphology and position of the disc, removal of the disc with or without replacement. [31]

\subsection{Physical Therapy}

Physical therapy is used by TMD patients to keep the synovial joint lubricated, and to maintain the jaw motion. One of the exercise for the jaw is to open the mouth to a comfortable fully-open position and then apply a slight additional pressure to open the mouth fully. Another exercise includes stretching the jaw muscles by doing various facial expressions. Avoiding extreme jaw movements, taking medications, applying moist heat or cold packs, eating soft foods are other ways that may keep the disorder from worsen.[5]

\subsection{Splints}

They are plastic mouthpieces that fit over the upper and lower teeth. They prevent the upper and lower teeth from coming together, lessen the effects of clenching or grinding the teeth. Bruxism also causes the TMJ dysfunction due to tooth attrition and subsequent malocclusion; myofascial strain, fatigue or fibrosis of masticatory muscles and capsulitis and adhesions within the TMJ joint space. Splints are effective in reducing the intensity of pain for patients with pain in jaw and masticatory muscles by compensating for or correcting perceived bite defects of the sufferer. The studies on evidence-based medicine for splint therapy, however, have shown equivocal results. The long-term effectiveness of the therapy has been widely debated and remains controversial. [5]

\subsection{Pharmacotherapy}

The pharmacologic agents which are commonly prescribed nonsteroidal anti-inflammatory drugs (NSAIDs) to reduce inflammation. However, research is needed to exploit long term use and to identify whether the advantages in the management of pain and inflammation outweigh the negative side effects. Muscle relaxants also be prescribed for treatment of muscle pain and spasm. However, studies have failed to demonstrate that muscle relaxants are any more effective for pain relief than NSAIDs. To increase their benefit, muscle relaxants combination with NSAIDs are used . [32] 


\subsection{Arthroscopy}

Lysis of adhesions and joint lavage are the most commonly performed TMJ arthroscopic surgical procedures to relieve painful hypo mobility. The objective is to eliminate restrictions on the disc and lateral capsule, to wash out micro debris resulting from the breakdown of the articular surfaces, to irrigate the joint by enzymes and prostaglandins and to stimulate the normal lubricating action of the synovial membrane. In addition, the presence of fibrous adhesions in the superior joint space limits normal translatory function of the disc condyle complex.However, the pathogenesis of adhesions remains unclear, suspected that a macro or micro trauma induces hemorrhage; in the presence of limited joint mobility, the blood clot that forms will organize into a fibrous adhesion. Generally, a blunt trocar or blunt probe is utilized in a sweeping fashion between the disc and temporal bone to accomplish lysis of adhesion.[33] In 1975, Ohnishi described the use of arthroscopy in the TMJ .. The physical action of lysis and lavage in the superior joint space, rather than disc repositioning, is believed to be responsible for the success of arthroscopic surgery in earlier studies. [34,35] This factor led to the use of TMJ arthrocentesis as relatively less invasive alternative to reduce the inflammation in the joint space and to restore the function.[34]

\subsection{Arthrocentesis}

Nitzan who decribed the arthrocentesis ,simplest and minimal invasive form of surgery in the TMJ, aim to release the articular disc and to remove adhesion between the disc surface and the mandibular fossa by means of hydraulic pressure from irrigation of the upper chamber of the TMJ. [36] This is very effective procedure in patients with persistent or chronic closed lock and anchorage in the upper articular space. Lavage of superior joint space with saline exerts its effects through its ability to eliminate joint effusion to reduce the symptoms. It is considered as an intervening treatment modality between nonsurgical treatment and arthroscopic surgery.[37]Being the least invasive and simplest form of surgical interventions into the TMJ, this procedure carries a very low risk and relatively easy to proceed in dental chair office under local anaesthesia alone or in combination with conscious sedation. [38]

\subsection{Discectomy And Disc Replacement}

Discectomy used to regain the mandibular motion and to reduce orofacial pain, and may be followed by disc replacement. It has been shown in 5- and 10-year postoperative follow-ups to increase mandibular motion in patients previously showing no improvement with non invasive management modality. Patient experience with disc replacement demonstrates the unanswered need for autologous tissue replacements that are capable of function in the complex loading environment of the TMJ, patients experiences continued joint degeneration need a functional, non pathogenic disc replacement. [32]

\subsection{Joint Reconstruction}

Several techniques have been proposed for reconstruction of portions of the joint or the entire joint itself. A hemiarthroplasty may be used to replace the superior articulating joint surface. During reconstruction, joint adhesions are lysed and a Vitallium alloy fossa-eminence prosthesis, manufactured by TMJ Implants, is implanted to replace the temporal component of the joint. Importantly, condylar change often accompanied by degenerative change in the temporal component of articulation. In this case, total joint reconstruction may be necessary. If immune-mediated processes are not present, a costochondral graft permits a comprehensive reconstructive option in which autologous costochondral segments replace the condyle with a biologic graft; as a native tissue, its inherent adaptability and lack of immunogenic potential offer significant advantages over alloplastic materials. Alloplastic alternatives appear to be better suited for the treatment of these patients and those with immune-mediated degenerative processes.[32] The currently available U.S. Food and Drug Administration- approved alloplastic total joint replacement systems include The Christensen Total Joint System (TMJ Implants), the TMJ Concepts System (TMJ Concepts), and the Biomet Microfixation prosthetic total joint (Biomet Microfixation). Implant lifetimes are in the range of 10 to 15 years; considering the average age of TMD patients, secondary surgery is often necessary. [32]

\subsection{Intra Articular Injection Of Cortico Steroid}

Intra articular injection of cortico steroids alone $[40,41]$ or after arthrocentesis provides long-term palliative effects on subjective symptoms and clinical signs of TMJ pain . Recently, Sodium Hyaluronate (SH) has been proposed as an alternative therapeutic agent which is high viscous, high molecular substance plays an important role in joint lubrication and protection of the cartilage.[39] Various studies have demonstrated the use of drugs like Morphine, Fentanyl, Bupivacaine, Corticosteroids and SH for the management of TMJ disorders. Corticosteroids have a potent anti inflammatory action on synovial tissue and well known to reduce effusion, decrease pain and brings about an increase in range of motion of synovial joints; $1 \mathrm{ml}$ of betamethasone is routinely used at the end of lysis and lavage of superior compartment of TMJ.[36 42] Hyaluronic acid (HA) is a 
normal product of joint tissues that is continuously released into the synovial fluid, where serves as a lubricant, anti inflammatory, and pain reliever and also acts as a adjunct. The most common visco supplementation strategy is a series of either two intra-articular HA injections spaced 7-14 days apart [44] or five injections each 7 days apart.[43] Several randomized comparisons of intra-articular hyaluronic acid (sodium hyaluronate) and corticosteroid (betamethasone). Intraarticular injection of sodium hyaluronate or corticosteroid (betamethasone) following arthrocentesis has a considerable effect. [45]

\subsection{Low Level Laser Therapy}

Clinical studies of LLLT used on patients with disc derangement disorders using either AlGaAs 830 $\mathrm{nm}$ diode laser in continuous wave mode or $\mathrm{He} \mathrm{Ne}$ laser $632 \mathrm{~nm}$ combined with a diode laser $904 \mathrm{~nm}$ in pulsed mode have shown clinical benefits in terms of reduction in pain and clicking. The application of laser beams diminishes pain while simultaneously reducing muscle contraction. When a local effect is desired, laser is effective by stimulating microcirculation and local cell tropism. When a general effect is needed by promoting pain relief for a variety of etiology, including irradiation of trigger points in myofacial pain, acting on tissue repair, reduction of edema and hyperaemia. Similarly, Bertolucci \& Grey in 1995 reported significant improvement in articular noise, limitation of oral opening and also in the masticatory function through reduction of muscle contraction and of intra articular inflammation by LLLT. [46] The main effects of laser light used in LLLT on tissue include: Analgesic, Biostimulating , Anti- inflammatory .Advantages includes aseptic, noninvasive, painless, nonpharmaceutical and reversible therapy, if used properly has no side effects.It has no postoperative discomfort. Disadvantage has been the high cost compared to the conventional therapies and the fast development in the field.[46]

\subsection{Current Progress In Tissue Engineering}

Currently, the repair and replacement of pathologic TMJ tissues remains an unmet need, and tissue engineering shows long-term promise for meeting this demand. This challenge may be met using tissue engineering techniques to produce joint components that have the ability to adapt to mechanical and chemical stimuli produced by functional articulation. Tissue engineering initiate attempting to recreate the native condylar cartilage follow a three-part approach that considers cell sourcing, biomaterials for scaffolding, and bioactive stimuli.[32]

The following sections will outline design objectives and current strategies for condylar as well as discal tissue engineering, as depicted as below.

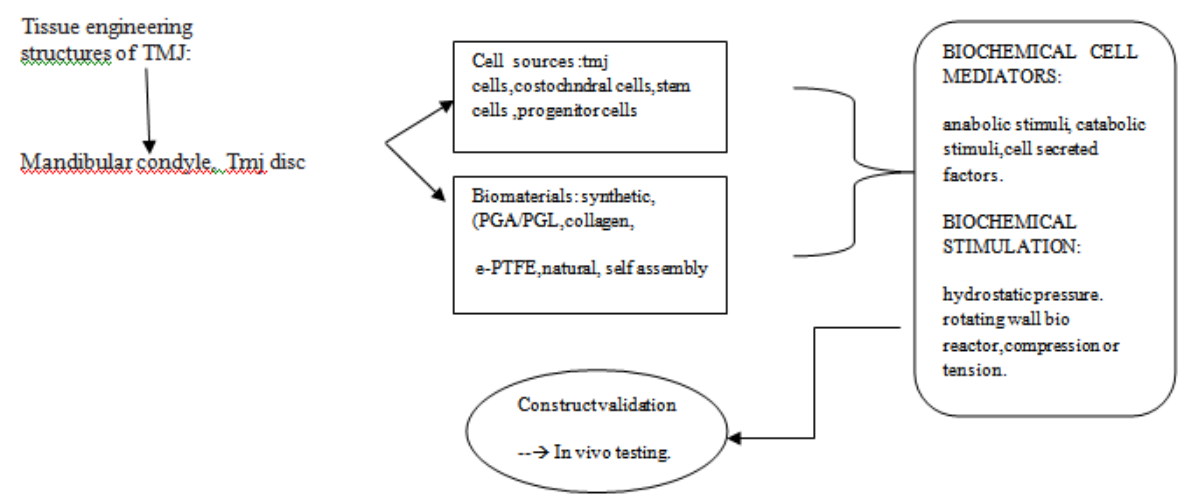

fig 3 Tmj tissue engineering strategy

It is important to note the significant donor site morbidity and potential pathology in TMD patients associated with this cell source. [32]

\subsection{Tissue Engineering Of The Tmj Disc}

Early studies exploring tissue engineering of the TMJ disc laid the foundation and demonstrated the potential effort , early work lacked the characterization information needed for validation and progress in optimizing the criteria of the designs. The disc shows biomechanical properties that may be matched more easily in tissue-engineered constructs in contrast to other musculoskeletal soft tissues. With respect to shear stimulation, shear stress is experienced in vivo by the disc during joint rotation and translation and may be simulated in culture via a rotating wall bioreactor. Another study seem to corroborate the results obtained by Nicodemus et al demonstrating the beneficial application of static over dynamic compressive loading for condylar tissue engineering.[32] Further investigation is needed to facilitate the potential independent benefits 
of mechanical stimulation and the interrelated benefits of both mechanical and biochemical stimuli for both disc and condylar cartilage tissue engineering.

\section{Conclusion}

Temporo mandibular disorders are frequent and wide spread in general population.TMJ internal derangement is most frequent type of TMD, and in characterised by several stages of dysfunction involving the condyle- disk relationship. The chief complaint is usually pain, which can manifest itself in different ways: head ache, jaw ache, ear ache, facial pain. In early stages of conditions, treatment may involve eating a soft diet and reducing strain on the jaw with the use of a splint or bite guard. Non steroidal anti-inflammatory drugs or muscle relaxants may be prescribed. Physical therapy and stress management may also be helpful in managing the condition. If the derangement becomes more severe or refractory to conservative and non - surgical treatment, it may be necessary to surgically repair ,reposition or possibly remove and consider replacing the disc or joint. demand .

Currently low level laser therapy and tissue engineering show long term promise for meeting this

\section{References}

[1] Dolwick MF, Katzberg RW, Helms CA,Internal derangements of the temporomandibular joint: fact or fiction?, J Prosthet Dent, 49, 1983, 415-8.

[2] Arati S. Neeli, Meenaxi Umarani, S. M. Kotrashetti, Shridhar Baliga, Arthrocentesis for the Treatment of Internal Derangement of the Temporomandibular Joint , J. Maxillofac. Oral Surg,9(4),(Sept-Dec 2010) ,350-354

[3] Farrar WB, Myofascial pain dysfunction syndrome, J Am Dent Assoc,102, 1981,10-1.

[4] Edvitar Leibur, Oksana Jagur and Ülle Voog-Oras , Temporomandibular Joint Arthroscopy, Dr Jason L. Dragoo (Ed.), Modern Arthroscopy,(Intech , 2011).

[5] Shirish ingawale and tarun goswami, temporomandibular joint: disorders, treatments, and biomechanics, Annals of biomedical engineering,37(5) , may 2009, 976-996.

[6] Wilkes C. H., Internal derangements of the temporomandibular joint, Arch. Otolaryngol. Head Neck Surg,115, $1989,469-477$.

[7] Gadd, A, and T. Goswami, Temporomandibular disorder and joint replacement, Biomed. Mater, 2009.

[8] Holmlund, A.B.\& Axelsson S, Temporomandibular arthropathy: correlation between clinical signs and symptoms and arthroscopic findings, International Journal of Oral \& Maxillofacial Surgery, 25(3), June 1996, 266-271.

[9] Emshoff, R, Clinical factors affecting the outcome of arthrocentesis and hydraulic distension of the temporomandibular joint, Oral Surgery Oral Medicine Oral Pathology Oral Radiology Endodontics, 100(4), October 2005,409-414.

[10] Voog, Ü,Alstergren, P, Eliasson, S, Leibur, E, Kallikorm, R. \& Kopp S, Inflammatory mediators and radiographic changes in temporomandibular joints in patients with rheumatoid arthritis, Acta Odontologica Scandinavica, 61(1), January 2003, 57-64.

[11] Voog, Ü, Alstergren, P, Eliasson, S, Leibur, E, Kallikorm, R. \& Kopp S, Progression of radiographic changes in the temporomandibular joints of patients with rheumatoid arthritis in relation to inflammatory markers and mediators in the blood, Acta Odontologica Scandinavica, 62(1), January 2004.

[12] Tallents, R.H, Macher, D.J, Kyrkanides, S, Katzberg, R.W. \& Moss, M.E.,Prevalence of missing posterior teeth and intraarticular temporomandibular disorders, Journal of Prosthetic Dentistry, 87(1), January 2002.

[13] Kim, S.J., Park, Y.H., Hong, S.P.,Cho, B.O., Park, J.W.\& Kim, S.G. , The presence of Bacteria in the synovial fluid of the temporomandibular joint and clinical significance: preliminary study, Journal of Oral and Maxillofacial Surgery, 61(10) ,October 2003, 1156-1161.

[14] Paegle, D.I., Holmlund, A.B., öStlund, M.R. \& Grillner, L. , The occurence of antibodies against Chlamydia species in patients with monoarthritis and chronic closed lock of the temporomandibular joint, Journal of Oral and Maxillofacial Surgery, 62(4), April 2004, 435-439.

[15] Leibur et al., 2010, Leibur, E., Jagur, O., Müürsepp, P.,Veede, L. \& Voog-Oras, Ü, Long-term evaluation of arthroscopic surgery with lysis and lavage of temporomandibular disorders, Journal of Cranio-Maxillo-Facial Surgery, 38(8), December 2010, 615-620.

[16] Alstergren, P., Kopp, S. \& Theordosson, E, Synovial fluid sampling from thetemporomandibular joint: sample quality criteria and levels of interleukin- 1 beta and serotonin, Acta Odontologica Scandinavica,57(1), January 2003, 278-282 .

[17] Kamada, A., Kakudo, K., Arika, T., Okazaki, J., Kano, M.\& Sakaki, T., Assay ofsynovial MMP-3 in temporomandibular joint diseases, Journal of Cranio-Maxillo- Facial Surgery, 28(3),June 2000,247-248.

[18] Alstergren, P. \& Kopp. S, Pain and synovial fluid concentration in arthritic temporomandibular joints, Pain , $72(1-2)$,August 1997.

[19] Voog, Ü.; Alstergren, P., Leibur, E.; Kallikorm, R. \& Kopp, S, Immediate effect of the serotonin antagonist granisetron on temporomandibular joint pain in patients with systemic inflammatory disorders, Life Sciences, 68(5), December 2000,591-602.

[20] Harms SE, Wilk RM, Wolford LM, et al, The temporomandibular Joint: magnetic resonance imaging using surface coils, Radiology ,157: 133, 1985.

[21] Katzberg RW, Bessette RW, Tallents RH et al, Normal and abnormaltemporomandibular joint: MR imaging with surface coil, Radiology, 158: 183, 1986

[22] Wilkes $\mathrm{CH}$, Arthrography of the temporomandibular joint in patients with the TMJ pain-dysfunction syndrome, Minn Med ,61:645, 1978 .

[23] Katzberg RW, Temporomandibular joint imaging, Radiology ,170: 297, 1989.

[24] Mercuri LG, Wolford LM, Sanders B. White RD et al,Custom CAD/CAM total joint reconstruction system: preliminary multicenter Repor, J. Oral Maxillofac Surg ,53:106, 1995.

[25] Sanchez-Woodworth RE, Tallents RH, Katzberg RW et al, Bilateral internal derangements of the temporomandibular joint: evaluation by magnetic resonance imaging, Oral Surg Oral Med Oral Path ,65: 281,1988.

[26] Schellhas K, Internal derangement of the temporomandibular joint: radiologic staging with clinical and pathologic correlation,Magnetic Resonance Imaging, 7 (5):495, 1989.

[27] Matteson SR et al, Bone scanning with technetium phosphate to assess condylar hyperplasia, Oral Surg Oral Med Oral Path, 60:356, 1985 . 
[28] Barkin S, Weinberg S , Internal derangements of the TMJ: the role of arthroscopic surgery and arthrocentesis, J Can Dent Assoc, 69,199-203.

[29] Goulet JP, Lavigne GJ, Lund JP ,Jaw pain prevalence among French speaking Canadians in Quebec and related symptoms of temporomandibular disorders, J Dent Res ,74, 1995,1738-1744.

[30] Mccarthy WL, Internal derangement of the temporomandibular joint,in Keith David A (Ed.), Surgery of temporomandibular joint, 2nd edn,(Boston : Blackwell Scientific Pub, 1992) 180-224.

[31] William A, Carvagal Daniel M, Laskin, Long term evaluation of arthrocentesis for the treatment of internal derangements of the temporomandibular joint, J Oral Maxillofac Surg ,58,2000, 852-855.

[32] Meghan K. Murphy, BE1/Regina F. MacBarb, BS,Mark E. Wong, DDS,Kyriacos A. Athanasiou, PhD, PE, Temporomandibular Disorders: A Review of Etiology, Clinical Management and Tissue Engineering Strategies, Oral Craniofac Tissue Eng ,1,2011, $205-226$.

[33] Samuel Barkin, DDS, Simon Weinberg, Internal Derangements of the Temporomandibular Joint: The Role of Arthroscopic Surgery and Arthrocentesis, J Can Dent Assoc,66, 2000,199-203.

[34] Franklin Dolwick M , Intra articular disc displacement part I: its questionable role in temporomandibular joint pathology, J Oral Maxillofac Surg, 53,1995,1069-1072

[35] David Frost E, Barry Kendell D , "The use of arthrocentesis for treatment of temporomandibular joint disorders", J Oral Maxillofac Surg ,57,1999,583-587.

[36] Nitzan DW, Dolwik MF, Martinez GA, Temporomandibular joint arthrocentesis: a simplified treatment for severe, limited mouth opening, J Oral Maxillofac Surg, 4, 1991,1163-1167.

[37] Frost DE, Kendell B ,The use of arthrocentesis for treatment of temporomandibular joint disorders, J Oral Maxillofac Surg ,57,1999, 583-587.

[38] Alpaslan C, Dolwick MF, Heft MW , Five year retrospective evaluation of temporomandibular joint arthrocentesis, Int J Oral Maxillofac Surg ,32,2003,263-267.

[39] Alpaslan GH, Alpaslan C, Efficacy of temporomandibular joint arthrocentesis with and without injection of sodium hyaluronate in the treatment of internal derangements, J Oral Maxillofac Surg ,59,2001,613-618.

[40] Koop S, Carlsson GE, Haraldson T, Wenneberg B , The short term effect of intra-articular injection of sodium hyaluronate and corticosteroid on temporomandibular joint pain and dysfunction, J Oral Maxillofac Surg, 43,1985,429-435.

[41] Koop S, Carlsson GE, Haraldson T, Wenneberg B ,The long term effect of intra-articular injection of sodium hyaluronate and corticosteroid on temporomandibular joint arthritis, J Oral Maxillofac Surg ,43, 1987,929-935.

[42] Nitzan DW, Dolwick MF, Heft MW , Arthroscopic lavage and lysis of the TMJ: a change in perspective, J Oral Maxillofac Surg, 48,1990,798-801.

[43] Guarda-Nardini L, Manfrendini D, Ferronato G, Shortterm effects of arthrocentesis plus viscosupplementation in the management of signs and symptoms of painful TMJ disc displacement with reduction. A pilot study, Oral Maxillofac Surg ,14,2010,29-34.

[44] Alpaslan C, Bilgihan A, Alpaslan GH, Güner B, Ozgür Yis M,Erbas, D, Effect of arthrocentesis and sodium hyaluronate injection on nitrite, nitrate, and thiobarbiturate acid-reactive substance levels in the synovial fluid,2000.

[45] Wenneberg B, Kopp S, Gro"ndahl HG , Long-term effect of intra-articular injections of a glucocorticosteroid into the TMJ: a clinical and radiographic 8-year follow-up, J Craniomandib Disord 5(1),1991,11-18.

[46] Khan, m, vijayalakshmi, k. r. \& gupta, n, Low intensity laser therapy in disc derangement disorders of temporomandibular joint : A review article, Int. J. Odontostomat., 7(2),2013,235-239. 